\title{
Educación para la vida: una alternativa para el desarrollo humano en Nicaragua y América Latina
}

\author{
Ernesto Alejandro Gómez Salazar. \\ Universidad Nacional Autónoma de Nicaragua, Managua \\ ernest.antropology@gmail.com
}

Recibido: agosto 2016. Aprobado: septiembre 2016

\section{RESUMEN}

El artículo de investigación "Educación para la vida: Una alternativa para el Desarrollo Humano en Nicaragua y América Latina" centra su interés en el análisis de la educación formal, no formal e informal como mecanismos de formación e integración sociocultural que constituyen las bases para el Desarrollo Humano. La complementariedad de la educación en sus diversos ámbitos es una realidad emergente para hacer de la educación "Otra educación posible" donde el ser humano sea el centro del proceso de enseñanza aprendizaje mediante la formación de valores culturales, la conciencia social y la necesidad eminente de replantear los paradigmas de la educación a bien de formar ciudadanos interculturales, con sentido de pertinencia y capacidad autocritica.

Palabras Claves: Educación para la vida; Pedagogía del oprimido; Desarrollo Humano; No sistema educativo

\section{ABSTRACT}

This research article "Education for Life: An Alternative to Human Development in Nicaragua and Latin America" focuses its interest in the analysis of formalTh, non-formal and informal training mechanisms and sociocultural integration that are the basis for the Human Development. The complementarity of education in their various fields is an emerging reality for education "Another possible education" where the human being is the center of the teaching-learning process through the formation of cultural values, social conscience and the imminent need for rethink the paradigms of learning good form intercultural citizens with a sense of relevance and self-critical capacity.

Key Words: Education for life; Pedagogy of the oppressed; Human development; In the education system

\section{INTRODUCCIÓN}

"Los hombres y las mujeres son productos de las circunstancias y de la educación, cuando ellos cambian se debe a otras circunstancias y a una educación modificada; por tanto, son los hombres y las mujeres que cambian las circunstancias y el propio educador necesita de educación". Karl Marx 
Los Gobiernos neoliberales y las políticas educativas en América Latina han incrementado las brechas de desigualdad, económica, social, política y cultural. En los países latinoamericanos es evidente el analfabetismo, la pobreza económica, la migración y la inseguridad ciudadana producida por la falta de atención del Estado y la familia a sectores excluidos por el sistema político - económico.

El modelo neoliberal conceptualizo la educación como el simple hecho de transmitir el conocimiento, designando así roles, docente - estudiante, esta acción limita el ejercicio educativo como medio para cultivar la capacidad de razonamiento y autocrítica de parte de los sujetos inmersos en esta práctica.

En países como Guatemala, El Salvador, Honduras, Nicaragua existen serios problemas referidos a la educación, producida por los bajos salarios de los docentes, agregado a ello, la falta de capacitación constante y la necesidad de innovar metodológica y tecnológicamente para transferir el conocimiento de forma asertiva.

Por esta razón, el mundo contemporáneo y la educación actual requiere reformular los paradigmas educativos, dinamizando roles entre docentes y discentes y a su vez, asumiendo responsabilidades entre la sociedad y el Estado, Juan Bautista Arríen concibe

la educación como proceso humano, social, cultural y económico en el que se activa todo su potencial, traducido en personas preparadas para compartir la vida, el bienestar y el desarrollo común, constituye una inmensa corriente que arrastra componentes originales previos muy importantes y genera la capacidad de engrosarlos cuantitativa y cualitativamente. (Arríen, 2012, p. 14).

Todo proceso educativo requiere centrarse en la condición humana desde una perspectiva de inclusión, pertinencia y calidad; a su vez, la práctica educativa implica una combinación de la dimensión sincrónica - diacrónica mediante el cual se comparten los saberes y experiencias acumuladas en relación a la cultura, el desarrollo económico, la historia, la tecnología y a su vez implica mayor acercamiento a las múltiples realidades que en muchos casos son denominadas "otras realidades" por ser desconocidas y finalmente forman parte de la misma realidad nacional vinculada a la multiculturalidad, a la plurinacionalidad y a establecer una clara diferenciación entre teoría y práctica e implementar la educación como proceso que trasciende el espacio formal de la escuela.

La teoría antropológica fundamenta el análisis de la educación, pero a su vez retoma aportes de teóricos y la experiencia de educadores con conocimiento en la materia, en particular, el maestro 
Miguel de Castilla quién fungió como ministro de educación durante el periodo de gobierno del Frente Sandinista, también se consideran los aportes del maestro Dolores Juliano y Paulo Freire, para fundamentar el análisis crítico de este trabajo.

Es evidente la asimetría social, política y cultural del sistema educativo formal con respecto a la realidad social de la sociedad nicaragüense, identificando así la necesidad urgente de implementar una política educativa de Estado Nación que articule los aportes de los diversos sectores de la educación formal e informal.

Es de interés analizar la importancia de los procesos de educación integral como estrategias de educación para la vida, así también promover la articulación de estrategias de educación formal, no formal e informal y finalmente reflexionar sobre la necesidad de implementar una educación para la vida, con sentido de pertinencia y respuesta a las problemáticas sociales latentes en la sociedad.

Comprender la realidad social del ser humano en sus diversos escenarios sociales, culturales, políticos, económicos y concebir de forma integral la vinculación existente entre los múltiples escenarios puede lograrse con mayor acercamiento mediante el método etnográfico de investigación propio de la ciencia antropológica.

Las unidades de análisis propuestas constituyen el punto de partida para crear una discusión teórica en base a la experiencia cotidiana en los procesos de educación, considerando las escuelas clásicas como son; Estructuralismo vinculado a la educación formal, funcionalismo vinculado a la educación no formal e informal, procesos de socialización, educación formal, no formal e informal y finalmente educación como práctica liberadora, propuesta por Paulo Freire.

\section{DESARROLLO}

Históricamente la educación se ha concebido como un proceso escolarizado dirigido desde las políticas de gobierno en correspondencia con fines y objetivos establecidos a través del Ministerio de Educación, el modelo educativo aplicado no ha trascendido más allá del aula, por esta razón la dinámica educativa se encuentra alejada de la realidad sociocultural, por esta razón es necesario articular la dinámica educativa y concebirla en la práctica cotidiana como mecanismo directo para dar respuesta a las demandas sociales existentes en la sociedad. 
Los Gobiernos de los países latinoamericanos establecen políticas educativas de acuerdo al gobierno de turno el cual trabaja en función de sus objetivos e intereses ideológicos, este se convierte en un sistema asimétrico, al cual Miguel de Castilla denomina el no sistema educativo nacional

El sistema educativo nacional, en estas circunstancias, se define como un 'no sistema' educativo nacional. No hay coherencia. No hay sistema. Hay fragmentos y caos en una función que al estar al servicio de las personas y la sociedad deberá ser armoniosa, articulada y sistémica, desde el inicio, cuando la persona nace, hasta el final, cuando la persona muere (Castilla de, 2005, p. 15).

La fragmentación del sistema educativo se evidencia en el modelo educativo convencional que históricamente se impulsó durante los gobiernos neoliberales para desarrollar sujetos pasivos, limitando su capacidad de movilización, actitud autocrítica para generar propuestas ante las realidades existentes que afectan a la colectividad.

Se ha demostrado que la educación es un proceso histórico que nace y se desarrolla en la sociedad, garantiza la supervivencia y el progreso, transmitiendo de una generación a otra, los conocimientos, valores, principios y hábitos. Así mismo, da a conocer los avances que se dan en la sociedad también orienta el futuro de ella. Este proceso incluye formación de individuos en las ideologías, en un complejo sistema de valores e ideas que justifican los intereses de la clase que controla el poder. (Blandón, 2004, p. 14).

El sistema educativo desarrollado es meramente un proceso estructural distante de las realidades sociales. La lejanía de la escuela con respecto a las demandas de la sociedad implica un estancamiento de la práctica educativa en Nicaragua y en muchas zonas rurales de Guatemala, El Salvador, Bolivia, Ecuador, Venezuela y el contexto latinoamericano, producido por la falta de visión colectiva que permita al Estado y la sociedad desarrollar estrategias como mecanismos de respuestas a problemas sociales y económicos en la esfera comunitaria.

El cambio de gobiernos implica rupturas en la aplicación del Plan Nacional de Educación integral, así lo plantea Blandón (2004):

El año 2000, se trabajó el Plan Nacional de Educación 2001 - 2015. Este se concibió con la intención de que la educación se formule de tal manera que no sufra de los cambios bruscos, según la filosofía de los gobiernos que se instalen consecutivamente en este periodo. Es el 
instrumento donde indican las tendencias fundamentales que deberá tener la educación nicaragüense en los próximos quince años. (p.16)

El Plan Nacional de Educación constituye una herramienta para desarrollar la política pública nicaragüense en el sector educativo. Sin embargo, la aplicación del plan se enfrenta a grandes retos, durante el cambio de los gobierno, dado que se formulan estrategias y políticas educativas de acuerdo a los fines y objetivos perseguidos por los funcionarios elegidos para cada periodo, por lo cual, el sistema educativo, carece de una política estrategica a largo plazo, esto afecta el impacto social de la educación en la sociedad nicaragüense.

Las politicas sociales y en particular la educativa debe tener como premisa fundamental cuatro principios identificados por Delors (1997): “Aprender a conocer, aprender a hacer, aprender a ser y aprender a convivir". El primero está basado en el conocimiento previo de la persona respecto a lo cotidiano, la ciencia y la cultura; el segundo concibe la educación como medio alternativo donde el discente desarrolla habilidades, capacidades y destrezas para la vida; el tercero implica la capacidad misma puesta en práctica para solventar su propia realidad en base a la identidad colectiva y la personalidad; el cuarto principio concibe la importancia del aprendizaje a través de las relaciones sociales, la práctica de valores, la convivencia pacífica y la interculturalidad como medio de intercambio social, político y educativo.

La transferencia de conocimientos es fundamental para el desarrollo humano del individuo con el fin de promover una educación para la vida, vinculando las realidades sociales de la familia, la comunidad, la escuela y el trabajo de formación realizado por organizaciones no gubernamentales en pro del desarrollo de las personas.

Toda la tarea de educar sólo es auténticamente humanista en la medida en que procure la integración del individuo a su realidad nacional, en la medida en que pierda el miedo a la libertad: en la medida en que pueda crear en el educando un proceso de recreación, de búsqueda, de independencia y a la vez de solidaridad (Paulo, 1965, p. 13).

Todos los actores de la sociedad asumen en algún momento de la vida el rol de educador, ya sea mediante la transferencia de la cultura y los valores, a sus hijos, nietos, hermanos, amigos, esta es concebida como educación informal, fundamentada en la socialización primaria cuyo seno de aplicación es la familia espacio en el cual se establecen relaciones sociales, intercambios culturales, aprendizajes colectivos e individuales. 
Para tener una educación más vinculante es necesario que él educador conciba el procesos de formación como dinámica constante asociada a la realidad cultural del grupo, por esta razón se considera necesario iniciar el análisis antropológico retomando a Harris (1996) quien concibe la cultura como elemento indisoluble de la comunidad, ya que esta, constituye la base de la sociedad. "La cultura hace referencia a las formas pautadas de pensar, sentir y comportarse de los miembros de una población". (Harris, 1996, p. 535)

El primer espacio de formación e interacción es la familia, en este particular, es la institución social base de toda sociedad donde se articulan las relaciones sociales y se construye la identidad del individuo en base a valores culturales, éticos, morales y en algunos casos asociado a los principios religiosos.

La construcción de la personalidad como elemento identitario del individuo responde a procesos de socialización primaria en la esfera de la familia y la comunidad Victor Barnouw (como cita por Harris, 1997) plantea: "La personalidad es una organización más o menos duradera de fuerzas dentro del individuo asociadas a un conjunto de valores, actitudes, y modos de percepción estables que explican la consistencia de la conducta del individuo". (p. 535)

La transmisión de la cultura es fundamental para la sociedad a bien de conservar viva sus raíces, costumbres y tradiciones, esta acción es posible a través de la tradición oral, por medio de la comunicación se transmiten códigos y pautas de conducta característicos de un individuo o colectividad. En esta dimensión es necesario plantear la importancia de la educación en la construcción colectiva. El autor Fromm (como cita Crawford, 1983) considera

El papel de la educación en la formación de los rasgos de la personalidad. Un elemento del proceso educacional es la familia la cual transmite e inculca al niño el espíritu de la sociedad. (...) en consecuencia, la familia puede considerarse el agente psicológico de la sociedad. (p. 35)

La consideración anterior se expresa en teoría, sin embargo en la aplicación práctica la familia tiene un gran nivel de disfuncionalidad producto de las prácticas globalizadas que el sistema dominante incentiva a través de los medios de comunicación y tecnológicos, se ha descuidado la formación primaria de padres a hijos en la escuela de la vida, la familia, la comunidad donde se requiere desarrollar principios afectivos. La educación en el seno primario es denominada educación informal. Los procesos no intencionales quedarían ubicados en el sector informal. También podemos destacar la Educación informal "se caracteriza por la conciencia del educado, es la educación producida con una 
acción cuya forma educativa no ha podido expresamente ser establecida para educar" (Bernet, 1996, p. 29)

A pesar de carecer de intencionalidad, la familia induce a los niños y niñas a la educación inicial, en este particular dependerá del educador la sistematicidad de la educación, su interacción con el educando, para desarrollar una educación integral en base a la dinámica de reflexión acción formando así sujetos autónomos con capacidad de reflexionar, criticar y proponer ante las diversas problemáticas de la sociedad.

Esta es la propuesta ideal de la práctica educativa en la familia, sin embargo, producto de la inexistencia de manuales o estrategias que contribuyan a un proceso de educación asertiva. Aún existen brechas en este espacio educativo, dado que los padres tienen mayor preocupación por la satisfacción de las necesidades materiales, sin embargo descuidan la parte afectiva y la comunicación, aspectos fundamentales en el crecimiento de toda persona.

La educación en la familia implica transferencia de conocimiento, muy pocos padres dedican el espacio para hablar con sus hijos durante la adolescencia en relación a los cambios físicos, sociales y actitudinales que vive el ser humano durante esa etapa. Este tema crea cierta confusión entre el adolescente, en muchos casos problemas de autoestima o bien limita la socialización con personas de su misma edad, en este caso lo más idóneo sería ver a los padres hablando sobre la transición del adolescente, hablar de sexualidad para el respetar y disfrutar de manera responsable.

El tema de la sexualidad es aún un tabú entre las familias de Centroamérica, sin embargo en países como España, Argentina, Venezuela existe mayor madurez en relación al tema.

La falta o poca atención de los padres contribuye a la búsqueda de información en otras partes, no siempre es la mejor alternativa, sin embargo incide en el comportamiento de los infantes, adolescentes y jóvenes. En algunos casos, la falta de atención ha tenido implicaciones graves como el consumo de sustancias alcohólicas y drogas de forma irresponsable, conllevando así la rebeldía, problemas de inseguridad ciudadana y familiar que trascienden a la esfera comunitaria.

Para prevenir situaciones como la mencionada anteriormente es fundamental que los padres dediquen tiempo a la convivencia y atención a sus hijos, de esta forma la familia crecerá en unidad y cohesión, disfrutando plenamente de las relaciones de género, la igualdad e inclusión social, a su vez el seguimiento escolar y reforzamiento son necesarios para cultivar el hábito de lectura e inducir al infante en la necesidad de participar en el procesos de forma autónoma. 
El siguiente paso es la educación formal, esta se encuentra dirigida desde el Estado

La educación formal, el hecho de que constituye una forma colectiva y presencial de enseñanza y aprendizajes; la definición de un espacio propio, (la escuela como lugar), el establecimiento de tiempos prefijados de actuación, la separación institucional de dos roles asimétricos y complementarios (maestro alumno) la preselección y ordenación de los contenidos que se trafican entre ambos por medio de planes de estudios y curricula. (Bernet, 1996, p. 29).

Históricamente la educación ha respondido a intereses políticos e ideológicos de los gobiernos de turno, según sean sus fines para formar políticamente a la sociedad, en algunos casos, limitar la educación a la escuela, sin fomentar una posición crítica por parte de los discentes, ejemplo preciso fue la política educativa impulsada por los gobiernos neoliberales al servicio de los sectores con mayores recursos económicos.

Poco es el presupuesto destinado a la educación en los países latinoamericanos donde los esfuerzos se han concentrado principalmente en la educación secundaria y Universidad. Sin embargo, es necesario mencionar la importancia de la educación técnica y vocacional, el joven que ingresa a la educación técnica adquiere habilidades a mediano plazo para su puesta en práctica, una vez finalizado el curso cuenta con mayores oportunidades para tener acceso a empleo digno, generando de esta manera la sostenibilidad económica del sujeto, quien al tener empleo, tendrá mayores oportunidades de profesionalización.

En países como Cuba, la educación es tema prioritario, poca o escasa es la población fuera del sistema de educación formal. Sin embargo, es necesario equiparar las oportunidades en relación a la demanda de empleo, este es un problema existente en américa latina y el mundo, a como se requiere invertir en educación. También se requiere destinar parte de la inversión pública y atraer la inversión extranjera para incrementar alternativas de empleo y auto empleo.

La educación se ha implementado de forma estructural, esto genera asimetría en la dialéctica de su naturaleza, por la cual, se han concebido los procesos educativos como mecanismos de formación y respuestas a problemáticas de la sociedad. Es importante una educación pertinente para promover el desarrollo personal de sujetos con principios, capaces de generar y dirigir acciones para dar respuesta a los problemas sociales existentes en las comunidades.

Para fomentar una sociedad autónoma con capacidad autogestionaria, se necesita articular la educación informal, formal y educación no formal. Según Bernet (1996) 
La educación no formal sería aquella que tiene lugar mediante procedimientos o instancias que rompen con alguna de estas determinaciones que caracterizan a la escuela. La educación a distancia sería no formal puesto que no es presencial y rompe con la definición de espacio y tiempo (...) la enseñanza preceptoral puesto que no es una forma colectiva de aprendizaje; o también un centro de intercambio cultural, ya que difumina la separación de roles (p. 29).

Es decir, muchos de los procesos de educación no formal están relacionados con intenciones de mejorar las prácticas de la vida, de promoción social de actitudes, valores y de diálogo social. Muchos de estos objetivos o ejes se basan en el aspecto del comportamiento humano, de desarrollo social, de entendimiento y de la colectividad, es una educación que en muchos de sus ejemplos y de sus prácticas; se trata de educación para la vida, para dar respuesta a necesidades de su comunidad, más allá de lo racional y lo teórico, se enfoca en lo humano, en lo práctico, en la intersubjetividad.

Tres casos específicos a mencionar en la región centroamericana. En Nicaragua la Asociación Centro Cultural Batahola Norte desde 1983 trabaja en función de la restitución de derechos mediante la metodología de educación popular y a su vez en coordinación con Centros Educativos regidos por la educación formal.

El caso de El Salvador un poco más complejo pero de interés para su estudio, la organización no gubernamental Homies Unidos trabajo durante más de 10 años en procesos de formación y reinserción social con jóvenes de las Maras, habilitando así un proceso permanente de consejería, a su vez, se les doto de medios para la generación de autoempleo.

La Asociación Grupo Ceiba de Guatemala asume un trabajo similar al de El Salvador, en este particular, brindan atención de forma integral, mediante el albergue a jóvenes en riesgo, capacitación tecnológica y la puesta en práctica de los conocimientos mediante las prácticas que permiten el posicionamiento de los jóvenes en el mercado laboral.

Los casos en mención muestran claramente los aportes de las organizaciones no gubernamentales a la educación formal, siendo este un proceso complementario para atender aquellos aspectos que la educación tradicional no retoma en los países de Latinoamérica; los casos en mención se profundizaran en el escrito "Educación popular en Centroamérica "Otra educación es posible".

\section{Impacto sociocultural de la educación}

Otra educación es posible mediante la incorporación de los elementos socioculturales que caracterizan al grupo social. Este aspecto es de profundo interés para el reconocimiento de las 
realidades en las cuales se encuentra la población educativa, a su vez, este proceso requiere acompañarse de un estudio exploratorio que permita al educador y actores externos vivir cultura, lo cual implica ser un miembro más de la colectividad con deberes y derechos que corresponden al grupo. Solamente así podrá visualizar la necesidad misma de hacer de la educación una realidad integradora de prácticas y estrategias pedagógicas que faciliten la comprensión de la dinámica educativa.

El modelo de educación no formal brinda aportes sustanciales en la formación de la población de comunidades urbanas y rurales donde se trabaja con los diversos grupos etarios en procesos de formación de liderazgo, autoestima, desarrollo personal, comunicación y cultura de paz. Esta acción genera cambios en la esfera individual y colectiva permitiendo implementar estrategias comunitarias mediante el dialogo y los intercambios comunitarios. Estos son la base para los procesos de mediación y resolución de conflictos donde los ancianos asumen el rol de mediadores y consejeros de su comunidad.

En el ámbito sociocultural, las acciones desarrolladas influyen en la vida de los habitantes de la localidad, cuando se habla de educación para la vida. Se pretende contribuir a desarrollar estrategias para articular la educación informal, formal y no formal como medios de respuestas ante las adversidades de la vida. Educación para la colectividad, para disminuir los índices de violencia en base a prácticas fraternas que contribuyan a generar un dialogo social. Está educación será una práctica integradora como mecanismo de respuesta a las necesidades reales de la población, por tanto es importante reflexionar, la importancia de articular los tres modelos de educación, lo cual, generara mayor impacto en la vida de las personas, este se muestra directa o indirectamente en la vida comunitaria a partir del cambio de actitud y pensamiento en estrecha vinculación con el desarrollo humano.

la necesidad de diseñar un nuevo contrato social, basado en el consenso, así nace el desarrollo sostenible. Un Desarrollo en el que ninguna parte de la sociedad crece en detrimento de las demás, ya que el progreso de una parte no es real, si no es respaldado por el progreso de las demás partes. (Castillo: 2011, p. 8).

El centro de toda política de desarrollo debe ser el ser humano, y su principal regla la inversión centrada en las personas, en el capital social existente en cada territorio, es así como surge la conceptualización de desarrollo humano.

La educación de hoy es la base para un nuevo modelo de desarrollo basado en una concepción que revisa de manera exhaustiva el lugar que debe ocupar el ser humano en la naturaleza, y en un fuerte 
sentido de la solidaridad. Una política educativa puede, por lo tanto, convertirse en fuerza impulsora del desarrollo económico, social cuando forma parte de una política de desarrollo que integre la realidad local, municipal, departamental, regional, nacional e internacional; por esta razón se considera pertinente integrar a los Pueblos Indígenas en la generación de propuestas para hacer de la educación un instrumento de cambio constante.

La Declaración de las Naciones Unidas sobre los derechos de los Pueblos indígenas expresa en su artículo 4.

Los pueblos indígenas tienen derecho a todos los niveles y formas de educación del Estado. Todos los pueblos indígenas también tienen este derecho y el derecho a establecer y controlar sus sistemas e instituciones docentes impartiendo educación en sus propios idiomas y en consonancia con sus métodos culturales de enseñanza y aprendizaje.

Este instrumento jurídico se retoma en la legislación nacional y la política educativa, mediante esta se hace posible en Nicaragua el sistema de educación autonómico. La educación como proceso incentiva a formar al ciudadano desde una perspectiva intercultural, esto requiere basarse en principios democráticos para el bienestar colectivo y el buen vivir del pueblo mediante la participación ciudadana en procesos de consulta y validación de políticas públicas sociales.

La educación es un derecho colectivo y a su vez es el eje estratégico para el desarrollo de la nación y la comprensión de los pueblos y culturas de Latinoamérica para garantizar la convivencia entre las naciones del mundo; esto implica contar con una educación pertinente y de calidad enfocada en la equidad, los valores y principios tanto sociales, políticos y éticos que contribuyan al bienestar social.

\section{Educación para la vida: Un reto para la sociedad, el Estado y las ONG}

Luego de plantear aspectos teóricos referidos al tema, es pertinente aterrizar el planteamiento referido a educación para la vida, considerando que la dialéctica de la vida cotidiana demanda sujetos autónomos con capacidad de dar respuesta a los problemas sociales, económicos, culturales, políticos en las diversas esferas de la sociedad.

La educación en general fue creada en el momento de la estabilización de los Estados Naciones, se le asignó como función prioritaria la formación de ciudadanos capaces de contribuir con su trabajo al bien público, sin embargo, esta acción no se ha aplicado en términos prácticos, creando divergencia entre la propuesta de formar ciudadanos y el ejercicio del mismo. 
La escuela debe concebirse como el espacio de formación y comprensión de múltiples realidades que se presentan en la vida.

Así la escuela, presentada como maestra de la vida, se transforma en la vida misma para sectores importantes de la población. Este proceso de escuela continuada implica un aumento de la oferta laboral. Al mismo tiempo cambian las expectativas sociales (...) la presión que sufre del medio para adaptarse a las nuevas realidades, ofrece la oportunidad de profundizar en el análisis de una función social. (Juliano, 1963, p. 19)

El modelo educaivo requiere ser reestructurado y operativizarse mediante la creación de la comisión interinstitucional de educación integradao por lideres comunitarios, pueblos indígenas, organizaciones no gubernamentales, Universidades y el gobierno mediante sus instancias de educación. Esta instancia dará seguimiento a la asignación presupuestaria y a la política educativa en función de concebir la educación como estimulo ante la vida, desarrollando capacidades de adaptación en el sujeto para generar espacios de análisis y discusión donde se generen propuestas de acción comunitaria para solventar los problemas comunitarios, por ende mejorar la calidad de vida.

Educar para la vida implica retos para el Estado, es por esta razón que el sujeto, inicia un proceso de resistencia para generar cambios graduales en el sistema de educación formal.

La teoría de la resistencia que rescata la posibilidad de generar nuevas propuestas de los distintos actores sociales: y del análisis institucional que subraya por una parte la inercia que ofrece la escuela a las demandas sociales. (Juliano, 1963, p. 21)

El planteamiento anterior se encuentra en estrecha vínculación a la reflexión de Paulo Freire respecto a la realidad social objetiva, a través de la cual se requiere desarrollar la condición más humana en el individuo con sentido de identidad, conciencia y compromiso con el desarrollo personal, familiar y de la comunidad.

La realidad social, objetiva, que no existe por casualidad sino como el producto de la acción de los hombres, tampoco se transforma por casualidad. Si los hombres son los productores de esta realidad y si ésta en la "inversión de la praxis", se vuelve sobre ellos y los condiciona, transformar la realidad opresora es tarea histórica, es la tarea de los hombres. (Freire, 1970, p. 48)

El modelo de educación para la vida requiere la asociación de elementos como teoría, acción, realidad y reflexión "una forma de percibir es que uno piense, entonces, después del pensamiento viene 
la acción y luego la realidad" (Jica, 2007, p. 10). La sociedad demanda del Estado y la educación de hoy mayor coherencia y vinculación con la realidad a través de la aplicación de una pedagogía más humanista, pasar de la pedagogía del oprimido a la práctica liberadora. Esta acción implica la deconstrucción ideológica del sistema opresor, pasar de la teoría a la práctica social fundada en la transformación. Otro elemento a considerar es, transformar la realidad opresora y llegar a la liberación social, desde el enfoque de desarrollo en base a la condición humana, es decir un desarrollo educativo desde la familia, la comunidad, la cultura, lo social, lo político, es decir dimensionar su integralidad.

En este sentido, la interculturalidad se convierte en estrategia de acción educativa, por contribuir al aprendizaje reciproco de las culturas, donde toma en cuenta el conocimiento, su valoración, entendimiento y la voluntad de interactuar y participar en un ambiente de horizontalidad, respeto y solidaridad en un espacio de diálogo y discusión.

Las Universidades también desempeñan un rol protagónico en este proceso, en particular, hacer mención a retomar la visión de las Universidades comunitarias con estrecha vinculación a los pueblos indígenas. Hooker, rectora de la Universidad de las Regiones Autónomas de la Costa Caribe Nicaragüense, considera el rol preponderante de la Universidad, al respecto plantea

las universidades deben contribuir a que los pueblos originarios y comunidades afro descendientes puedan ejercer sus derechos a practicar y revitalizar sus tradiciones orales, filosofías, sistemas de escritura y literaturas, debiendo el Estado proporcionar los medios necesarios para tal cometido. (Hooker, 2008, p. 15)

En este sentido, la educación como estrategia de desarrollo estará orientada a la incorporar al sistema de educación a los sectores más vulnerables para incrementar el potencial profesional, la competitividad y la inclusión social, es de esta manera que el conocimiento se convierte en insumo fundamental del proceso productivo y la inversión en el capital humano es "más que nunca" necesaria para el progreso tecnológico, la competitividad y el crecimiento.

Nicaragua al igual que América Latina es una región con grandes riquezas sociales, culturales y ambientales, la Conferencia Regional de la Educación superior en América Latina y el Caribe destaca este aspecto "Nuestra región es marcadamente pluricultural y multilingüe. La integración regional y el abordaje 8 de los desafíos que enfrentan nuestros pueblos requieren enfoques propios que valoren nuestra diversidad humana y natural como nuestra principal riqueza" (CRES, 2008). 
Por esta razón es de urgencia implementar un nuevo paradigma de la educación enfocada en la integralidad, es decir en formar ciudadanos preparados para la vida. Este nuevo paradigma permitirá el empoderamiento de los pueblos y culturas del mundo, tomando en cuenta las enseñanzas de las generaciones ancestrales las cueles han tenido como base sus propias historias que forman parte de las lecciones de vida, la sistematización de buenas prácticas e intercambio lingüístico.

A su vez se requiere invertir en medios de formación profesional, promover la participación en programas de educación integral. Así también, los gobiernos deberán asegurar la disposición de recursos financieros, humanos y tecnológicos para hacer posible la ejecución de programas de educación intercultural; este modelo de educación deberá tomar en cuenta el entorno socio económico, cultural y las necesidades concretas de las poblaciones en su sentido más amplio y diverso.

A su vez la educación para la vida tendrá como fundamento indispensable la formación de ciudadanos comprometidos con su propia realidad y con las denominadas otras realidades, "Educar para la ciudadanía; asumir y respetar la dignidad del otro en tanto cualidad que responde a la identidad de cada persona, pues ésta se realiza cuando elige, cuando hace efectiva su libertad; aceptar la igualdad como la base fundamental de la convivencia social traducida en derechos y responsabilidades compartidas (Arríen, 2012, p. 18).

La educación como responsabilidad compartida por la sociedad, el Estado, las organizaciones no gubernamentales y las universidades requiere la aplicación de un modelo que combine docencia, extensión e investigación. Para acercar a los estudiantes desde la educación impartida en el aula a su realidad objetiva; esto incentivara a los discentes a participar en la vida comunitaria, tomando como base el modelo de participación ciudadana para emprender la ciudadanía intercultural, la autogestión comunitaria y el empoderamiento de los sujetos para gozar del derecho pleno a la autonomía y de esta forma transformar la sociedad a la cual pertenece.

Las propuestas referidas con anterioridad vendrán a dinamizar la educación por ende el desarrollo humano, para ello, se requiere voluntades políticas, sociales, culturales y económicas para generar sinergia y visualizar la complementariedad de los sistemas y el no sistema educativo para concebir y hacer de la educación un mecanismo para comprender las múltiples esferas de la vida cotidiana y de esta manera mejorar la calidad de vida de generación actual y las futuras generaciones. 


\section{REFERENCIAS}

Arrien, J. (2012). Las triadas de la educación, desde la persona. Breve ensayo para la reflexión. Instituto de Educación de la Universidad Centroamericana (IDEUCA - UCA). Managua, Nicaragua.

Bernet, J. (1996). La educación fuera de la escuela. Barcelona: Editorial Ariel. S.A.

Blandón, J. (2004). Políticas educativas en Nicaragua y rasgos de la educación general. Primer seminario internacional: La calidad y equidad de la educación”. León, Nicaragua: Universidad Nacional Autónoma de Nicaragua.

Castilla, M. (7 de Agosto de 2005). "El "no sistema" educativo nacional". Sobre la estructura del sistema educativo.

Castillo, L. (2011). Políticas y Estrategias Educativas para el Desarrollo Nacional. Seminario Nacional de Educación y Desarrollo. Managua, Junio 2011.

Conferencia Regional de Educación Superior de América Latina y el Caribe -CRES-. (2008). Celebrada del 4 al 6 de junio en la Ciudad de Cartagena de Indias, Colombia.

Crawford, J. (1983). Antropología Psicológica. El estudio de la personalidad en la cultura. Cuadernos de Antropología. Barcelona: Editorial Antrhopos.

Declaración de las Naciones Unidas sobre los Derechos de los Pueblos Indígenas. (2007). 107ª , sesión plenaria del 13 de septiembre.

Delors, J. (1997). La Educación encierra un Tesoro. México, D.F

Freire, P. (1970). Pedagogía del oprimido. Argentina: Argentina Editores S.A.

Harris, M. (1996). Introducción a la Antropología General. Madrid: Editorial Madrid.

Hooker, A. (2008). Educación superior y ciencia y tecnología en América Latina y el Caribe: Respuestas frente a la expansión y a la diversificación. Ponencia. I Congreso de Educación Superior en Nicaragua

JICA y UNAG. (2007). Taller de Capacitación, Metodologías de Motivación y Organización. Managua, Nicaragua.

Juliano, D. (1963). Educación Intercultural. Escuela y Minorías etnicas”. Madrid: Endema.

Paulo, F. (1965). La educación como práctica de la libertad. Argentina: Argentina Editores S.A

Sen, A. (1996). Capacidad y bienestar: En Calidad de vida. México, FCE. P. 54 